\title{
Renal Tubular Acidosis (RTA) due to Sjögren's Syndrome Presenting as Hypokalemic Quadriparesis - A Case Report
}

\author{
Choudary Annie Sheeba*, Sushanta Kumar Das, Sharadha Radhakrishnan, Konde Abbulu \\ Department of Pharm. D, CMR College of Pharmacy, Kandlakoya, Hyderabad, Telangana, INDIA.
}

\begin{abstract}
Sjögren's syndrome is a systemic autoimmune disorder that affects the exocrine glands and characterized by the development of lympho-plasma cell infiltrate, which causes progressive loss of glandular function. Primary form involves the exocrine glands, with or without systemic involvement; and also, a secondary form, which is associated with other autoimmune diseases e.g. rheumatoid arthritis, lupus erythematosus, scleroderma, inflammatory vascular and connective tissue diseases etc. Overt or latent Renal Tubular Acidosis (RTA) caused by tubule-interstitial nephropathy is a common extra-glandular manifestation of Primary Sjögren's Syndrome (PSS). PSS is most frequently diagnosed in female (female: male ratio of 9:1) and mostly diagnosed based on the results of salivary and/or lachrymal gland biopsies, examination of oral cavity and eyes and autoantibody assays. Here we present a case of 38 years old female patient with hypokalemic quadriparesis secondary to PSS.
\end{abstract}

Key words: Sjögren's syndrome, Autoimmune Disorder, Renal Tubular Acidosis, Tubule-interstitial nephropathy, Hypokalemic Quadriparesis.

\section{INTRODUCTION}

Sjögren's Syndrome (Sicca Syndrome) is a systemic chronic inflammatory disorder characterized by lymphocytic and plasmacytic infiltrates in exocrine organs, such as salivary, parotid and lachrymal glands. Non-exocrine organs like kidneys are often affected in Sjögren's syndrome; Distal Renal Tubular Acidosis (DRTA) and interstitial nephritis are common clinical condition in patients with Sjögren's syndrome. Nonetheless patients with Sjögren's syndrome rarely present with severe hypokalemia or paralysis secondary to DRTA. ${ }^{1}$ Although renal tubular acidosis with hypokalemia associated with Sjögren's syndrome has been reported earlier but hypokalemic quadriparesis as the initial manifestation of the disease are rare. ${ }^{2}$ Primary form involves the exocrine glands, with or without systemic involvement; there is also a secondary form, which is associated with other autoimmune diseases (rheumatoid arthritis, lupus erythematosus, scleroderma, inflammatory vascular and connective tissue diseases, etc.). ${ }^{3}$ The renal manifestations are related to tubular dysfunction resulting from chronic interstitial nephritis and can exhibit as DRTA, proximal renal tubular acidosis, tubular proteinuria and nephrogenic diabetes insipidus. Hypokalemic paralysis rarely occurs as the first manifestation of a renal tubule disorder due to PSS. ${ }^{4}$

Numerous criteria were proposed for diagnosis of Sjögren's syndrome amongst those most widely accepted are international classification criteria for Sjögren's syndrome developed by American and European group. These criteria include six different parameters:

\section{Ocular Symptoms (at least one) \\ Dry eyes $>3$ months \\ Foreign body sensation in the eyes \\ Use of artificial tears $>3 x$ per day}

DOI: 10.5530/ijopp.12.2.30

Address for correspondence: Ms. Choudary Annie Sheeba, Department of Pharm. D, CMR College of Pharmacy, Kandlakoya, Hyderabad-501401, Telangana, INDIA. Phone no: +91-9100384422 Email: anniesheeba. chowdary12@gmail.com

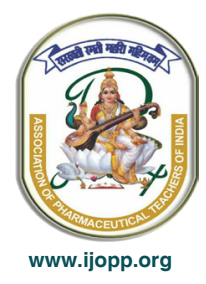




\section{Oral Symptoms (at least one)}

Dry mouth $>3$ months

Recurrent or persistently swollen salivary glands

Need liquids to swallow dry foods

\section{Ocular Signs (at least one)}

Schirmer's test, (without anaesthesia) $\leq 5 \mathrm{~mm} / 5 \mathrm{~min}$

Positive vital dye staining (van Bijsterveld $\geq 4$ )

\section{Histopathology}

Lip biopsy showing focal lymphocytic sialoadenitis (focus score $\geq 1$ per $4 \mathrm{~mm}^{2}$ )

\section{Oral Signs (at least one)}

Un-stimulated whole salivary flow ( $\leq 1.5 \mathrm{~mL}$ in $15 \mathrm{~min})$

Abnormal parotid sialography

Abnormal salivary scintigraphy

\section{Auto-antibodies (at least one)}

Anti-SSA (Ro) or Anti-SSB (La)

For a primary Sjögren's diagnosis: Any 4 of the 6 criteria must include either item 4 (Histopathology) or 6 (Auto-antibodies) or any 3 of the 4 objective criteria $(3,4,5,6)$.

For a secondary Sjögren's diagnosis: In patients with another well-defined major connective tissue disease, the presence of one symptom ( 1 or 2 ) plus 2 of the 3 objective criteria $(3,4,5,6)$ is indicative of secondary Sjögren's.,

Here we report a case of 38 years old female with Renal Tubular Acidosis due to Sjögren's syndrome presenting as hypokalemic quadriparesis.

\section{CASE PRESENTATION}

A 38 years old female patient was admitted in the Department of General Medicine on 2/8/2018 with the complaints of $(\mathrm{C} / \mathrm{O})$ multiple site body pains and weakness of all 4 limbs associated with inability to walk. On clinical examination $(\mathrm{O} / \mathrm{E})$ her vitals were found to be stable with slightly increased pulse rate ( 96 beats $/ \mathrm{min}$ ); she was unable to walk and her movement was witnessed to be slow.

History of Patient's Present Illness (HPI) enumerate about pain in lumbar region since last 4 years along with difficulty in night vision since 3 years.
Her past medical history reveals decreased appetite, weight loss, difficulty in swallowing, decreased saliva production since 2 years. She was also found to be positive for Anti Nuclear Antibody (ANA) profile and positive for RTA 1 year back. She was on Tab. Prednisolone $10 \mathrm{mg}$ BD but found to be medicine defaulter. Her day wise progress chart and prescription is listed in Table 1. Ophthalmologist report listed in Table 2 and various diagnostic tests were also performed throughout the hospital stay listed in Table 3.

\section{DISCUSSION}

Renal Tubular Acidosis (RTA) is a disorder of renal acidification out of proportion to the reduction in glomerular filtration rate characterized by normal Anion Gap (AG) metabolic acidosis. Type 1 RTA can cause hypokalemia; however, initial presentation of type1 RTA with hypokalemic quadriparesis with bulbar weakness and cardiac arrhythmia is extremely rare. In this case, patient is a known case d-RTA (diagnosed 1yr back) and now presented primarily with dysfunction of all the four limbs (quadriparesis). Only 14 such cases of distal RTA with hypokalemic muscle paralysis have been previously reported in association with Sjögren's syndrome. ${ }^{7}$

In this case; ocular symptoms, oral symptoms and positive ANA profile satisfies the diagnosis of Sjögren's syndrome. Biochemical investigations showed severe hypokalemia with hyperchloremic metabolic acidosis diagnosed as distal renal tubular acidosis. ${ }^{8}$ Association between the distal RTA with Sjögren's syndrome is quite frequent due the effect on exocrine glands (kidney). ${ }^{1}$

Management of pSS is symptomatic. In acute or severe conditions, especially when patient is present with hypokalemia, the priority will be to reverse severe hypokalemia by administration of IV Potassium supplementation, followed by correction of the underlying acidosis. Continuous usage of potassium supplementation might be required for majority of the patients. Use of muscarinic agonists (pilocarpine hydrochloride and cevimeline hydrochloride) is recommended for the treatment of oral dryness and, to a lesser extent, ocular dryness. Neuropathic pain in patients with primary Sjögren's syndrome is typically treated with gabapentin, pregabalin or duloxetine. Although no immune-modulators has proved to be efficacious in pSS, combination of corticosteroids and other immunosuppressive drugs has been reported to slow the progression of renal damage in Sjögren's syndrome. Agents that are commonly used include hydroxychloroquine, prednisone, methotrexate, 


\section{Table 1: Day wise progress and prescription.}

Day

Drugs and formulation

Upon admission she was prescribed with

Day 1

$(2 / 8 / 2018)$

admission

On day-6

$(7 / 8 / 18)$

On day-10

$(11 / 8 / 18)$

On day-11

$(12 / 8 / 18)$

On day-14

$(15 / 8 / 18)$

On day-17

$(18 / 8 / 18)$

On day-20

$(21 / 8 / 18)$

On day-21

$(22 / 8 / 18)$

Discharge
Tab. Calcium

Tab. Ranitidine

Tab. Multivitamine

patient was prescribed with

Inj. $\mathrm{HCO}_{3}$ 1-amp (7.5\%) Na-bicarbonate $=44.6 \mathrm{mEq} \mathrm{HCO}$ )

Inj. Pantoprazole

Tab. Calcium

Tab. Multivitamine

Tab. Vitamin- D3

Syp. Potassium Chloride $5 \mathrm{ml}(40 \mathrm{mEq} / 15 \mathrm{~mL})$

Tab. Nodosis ( $\mathrm{HCO}_{3}$ tablet)

Same treatment was continued up to day-5 (4/8/18 to 6/8/18)

Previous day prescription was continued with addition of

Syp. Potasium Citrate $5 \mathrm{ml}(5 \mathrm{mEq})$

Inj. Tramadol 50mg in 1 pint $(1 \mathrm{pint}=500 \mathrm{ml})$

Normal Saline

Syp. Potassium Chloride was Discontinuation and same medication continued up to day-9 (8/8/18 - 10/8/18)

She was referred to ophthalmologist and diagnosed with Dry Eye (Table-2) and was further prescribed with:

Eye Oint. Lacrigel

(Hydroxypropyl Methylcellulose)

Eye Drop.LU Mellose (carboxy methylcellulose)

$\mathrm{BD}$

Other medications were continued along with

Due to complaint of dry cough and due to her poor $\mathrm{Hb}$ value she was prescribed with two new medicines along with existing medicines

Inj. Augmentin (Amoxycillin + Clavulnic acid)

Tab. Iron Folic Acid $(100+1.5 \mathrm{mg})$

1.2gm IV BD

p/o BD

Same medication continued for day-12 (13/8/18) and day-13(14-8-18)

With fresh complaint of cough with mucoid sputum.

Syp. Ascoril (bromhexine, guaifenesine, menthol and terbutaline) $5 \mathrm{ml}$ BD Was added.

Same medication was continued for next two days.

new complaint of chest tightness was reported by the patient and

Tab. Taxim (cefotaxime). Same

$200 \mathrm{mg}$ p/o BD was added

Treatment was continued up to day-19 (20/8/18).

Tab. Prednisolone 40mg

p/o OD was added into

She was referred to orthopedic for fracture of right femur shaft and was prescribed with Inj. Zoledronic acid $5 \mathrm{mg}$ IV OD over $15 \mathrm{~min}$ slow administration.

Patient was discharged on with following discharge medicine for and was advised to review after 10 days.

Tab. Nodosis $\left(\mathrm{Na}-\mathrm{HCO}_{3}\right) 500 \mathrm{mg}$

Syp. Potchlor $5 \mathrm{ml}(15 \mathrm{ml}=20 \mathrm{meq}$ of $\mathrm{K})$

Tab. Vit-D3 (1000mg)

Calcium $(500 \mathrm{mg})$

Tab. Multi-vitamine (high levels of vit-B, vit-C and small amounts of Vitamin A, D, E)

T. Iron Folic Acid $100 \mathrm{mg}+1.5 \mathrm{mg}$

Syp. Ascoril

Inj. Tramadol 50mg

Inj. Pan (Pantoprazole)

Inj. Augmentin (Amoxicillin-1g + Clavulanate potassium-200mg) p/o TID

in half glass of water TID

p/o OD

$\mathrm{p} / \mathrm{o}$ OD

$\mathrm{p} / \mathrm{O}$ OD

p/o OD

$5 \mathrm{~mL} B D$

1 amp in 1 pint NS IV OD

40mg IV OD x 6days

1.2gm IV BD $x$ 6days 
Table 2: Ophthalmologist report.

\begin{tabular}{ccc} 
Eye Examination parameters & Right Eye & Left Eye \\
\hline Lids & Trichiasis + & Normal \\
Cornea & Quiet & Quiet \\
Pupils & Inferior epithelial defects+ & Inferior epithelial defects \\
Lens & NSRL & NSRL \\
Fundas & Normal & Normal \\
\hline
\end{tabular}

\section{Table 3: Various diagnostic tests throughout hospital stay.}

\section{Parameter}

ABG (3/8/18)

$\mathrm{pH}$

$\mathrm{pCO}_{2}$

$\mathrm{pO}_{2}$

$\mathrm{HCO}_{3}$

Serum Electrolytes (3/8/18)

Sr. Sodium

Sr. Potassium

Sr. Chloride

LFT and RFT (3/8/18)

Total Serum Bilirubin

Direct Bilirubin

Alkaline Phosphatase

Sr. Creatinine

Blood Urea

RBS

Complete Blood Picture (3/8/18)

Hemoglobin

RBC $\quad 8.8$

WBC 3.13

Platelets $\quad 5.88$

Neutrophils 281

Lymphocytes $\quad 68.4 \%$

Monocytes $\quad 21.9 \%$

Eosinophils $\quad 6.1 \%$

Basophils $3.1 \%$

$0.5 \%$

Strongly positive+++

Strongly positive +++

Positive ++

$14.43 \mathrm{mmol} / \mathrm{L}$

Sinus Rhythm, Left Ventricular Hypertrophy Anterolateral

2D-ECHO (7/8/18)

X-Ray (16/8/18)

Complete Urine Examination (17/8/18)

Spirometry $(20 / 8 / 18)$
$\mathrm{HR}-86 / \mathrm{M}$

ST-T

abnormality (due to LVH)

ECG (4/8/18)

Global hypokinesia of LV Mild LV systolic Dysfunction,

$$
\text { EF - } 42 \%
$$

\# proximal shaft of femur

Albumin: ++

Moderate restrictions
Normal Range

7.35-7.45

$32-45 \mathrm{mmHg}$

$75-100 \mathrm{mmHg}$

22-26mmol/L

$135-150 \mathrm{mEq} / \mathrm{L}$

3.5-5.0mEq/L

$96-106 \mathrm{mEq} / \mathrm{L}$

$0.2-1 \mathrm{mg} \%$

28-111/U/L

$0.6-1.2 \mathrm{mg} / \mathrm{dL}$

$15-40 \mathrm{mg} / \mathrm{dL}$

$70-110 \mathrm{mg} / \mathrm{dL}$

$12-15 \mathrm{gm} / \mathrm{dL}$

3.8-4.8mIn/cumm

4-11 Th. cells/cumm

$150-400 \mathrm{~L} /$ cumm

$40-80 \%$

$20-40 \%$

$2-10 \%$

$0-6 \%$

$0-2 \%$

$22-29 \mathrm{mmol} / \mathrm{L}$

mycophenolate sodium, azathioprine and cyclosporine. ${ }^{9}$ Similar management strategy was approached in this very case with almost matching the standard or recommended guidelines needed to manage this type of cases with addition of potassium supplementation. Furthermore, alkali supplementation was given as the supportive Indian Journal of Pharmacy Practice, Vol 12, Issue 2, Apr-Jun, 2019 therapy. Tramadol was preferred for pain management and prednisolone was added as the immunosuppressant. Zolendronic acid, a medication used to treat number of bone diseases was also prescribed to prevent the painful and easily broken bones. Hydroxypropyl Methylcellulose and carboxy methylcellulose was added to correct the dry 
eye condition.

\section{CONCLUSION}

Sjögren's syndrome is a systemic autoimmune disease that affects the exocrine glands. Middle aged female who present with hypokalemia and metabolic acidosis should be highlighted as high index of suspicion for possibility of Sjögren's syndrome. The case under study could have misled the physician as hypokalemia induced paralysis. But the supportive clinical features and positive antibodies coupled with keen observation along with laboratory data helped in the diagnosis of the condition. The confirmation of diagnosis has been done based on 4 criteria out the 6 standard criteria. The case was well managed with appropriate guidelines followed medication. Identification and diagnosis of this kind of clinical condition is not always clear and consistent. Hence, awareness of varied forms of presentation of this gradually progressive disorder should be encouraged. Clinical pharmacist must also be aware of these rare syndromes and support the clinicians in whatever capacity required. Far outreach to all healthcare professional in the form of such case studies can also be an additional tool to create awareness.

\section{ACKNOWLEDGEMENT}

We whole heartedly convey our sincere regards to the entire medical and non-medical staff of the Department of General Medicine, who has helped us during the entire period of case collection and discussion.

\section{CONFLICT OF INTEREST}

The authors declare no conflict of interest.

\section{ABBREVIATIONS}

ANA: Anti-Nuclear Antibody; Tab: Tablet; Inj: Injection; Syp: Syrup; Oint: Ointment; OD: Once a day; BD: Twice a day; TID: Thrice a day; QID: Four times a day; p/o: Per oral, IV: Intravenous; amp: Ampoule; mg: Miiligram; gm: Gram; mm: Milli meter; mL: Millilitre; d/L: Decilitre; L: Litre; mEq: Milli equivalent; mmol: Milli moles; IU: International Units; NS: Normal Saline; Hb: Hemoglobin; ABG: Arterial Blood Gas; ECG: Electrocardiogram; RFT: Renal Function Test; LFT: Liver Function Test; HR: Heart Rate; LVH: Left Ventricular Hypertrophy; EF: Ejection Fraction; RBS: Random Blood Sugar; Sr.: Serum; min: Minute; mmHG: Millimetre of mercury; $\mathrm{mln} /$ cumm: Million/SUMMARY
Middle aged female who present with hypokalemia and metabolic acidosis should be highlighted as high index of suspicion for possibility of Sjögren's syndrome. Supportive clinical features and positive antibodies coupled with keen observation along with laboratory data are significant in the diagnosis of the condition. Awareness of varied forms of presentation of this gradually progressive disorder should be encouraged. Clinical pharmacist must also be aware of these rare syndromes and support the clinicians in whatever capacity required. Far outreach to all healthcare professional in the form of such case studies can also be an additional tool to create awareness.

\section{REFERENCES}

1. Sengul E, Bunul F, Yazici A, Sengul A, Dindar S, Halhalli GS, et al. An Unusual Initial Presentation of Sjogren's Syndrome: Severe Hypokalemic Paralysis Secondary to Distal Renal Tubular Acidosis. Eurasian J Med. 2013;45(3):218-21.

2. Sarah S, Lijo G, Sukanya E, Rajasekaran D. Renal tubular acidosis due to Sjogren's syndrome presenting as hypokalemic quadriparesis: A report of two cases. Indian J Nephrol. 2015;25(6):386-7.

3. Dellafiore C, Villa A, Zibera F. Sjogren syndrome: A case report. Journal of Ultrasound. 2012;15(2):108-10.

4. Khandelwal D, Bhattacharya S, Khadgawat R, Kaur S, Tandon N, Ammini AC. Hypokalemic paralysis as a presenting manifestation of primary Sjögren's syndrome: A report of two cases. Indian J Endocrinol Metab. 2012;16(5):853-5.

5. Vitali C, Bombardieri S, Jonsson R, Moutsopoulos HM, Alexander EL, Carsons $\mathrm{SE}$, et al. Classification criteria for Sjögren's syndrome: A revised version of the European criteria proposed by the American-European Consensus Group. Ann Rheum Dis. 2002;61(6):554-8.

6. Jadhav S, Jadhav A, Thopte S, Marathe S, Vhathakar P, Chivte P, et al. Sjogren's Syndrome: A Case Study. J Int Oral Health. 2015;7(3):72-4.

7. Palkar AV, Pillai S, Rajadhyaksha GC. Hypokalemic quadriparesis in Sjogren syndrome. Indian J Nephrol. 2011;21(3):191-3.

8. Aygen B, Dursun FE, Dogukan A, Ozercan IH, Celiker H. Hypokalemic quadriparesis associated with renal tubular acidosis in a patient with Sjogren's syndrome. Clin Nephrol. 2008;69(4):306-9.

9. Sedhain A, Acharya K, Sharma A, Khan A, Adhikari S. Renal Tubular Acidosis and Hypokalemic Paralysis as a First Presentation of Primary Sjogren's Syndrome. Case Rep Nephrol. 2018;2018(10):1-4. https://doi. org/10.1155/2018/9847826 